nen Inaktivierungsquerschnitts $\sigma(T)$ mit den geometrischen Abmessungen der Phagen-DNS gezogen werden (vgl. Tab. 1 letzte Spalte). Bei einem Energieaufwand von $60 \mathrm{eV}$ pro Ionenpaar ${ }^{21}$ berechnet man aus unseren Versuchen im Mittel 5 Primärionisationen für die $\Phi \mathrm{X}$-DNS und 15 für die T1DNS pro Protonendurchgang. Die bei Zimmertemperatur mit Protonen gemessenen Inaktivierungsquerschnitte von $\Phi \mathrm{X}-174$ liegen nach der Korrektur auf die endliche Ionisierungsdichte zufällig nahe am Wert für den mittleren geometrischen DNS-Querschnitt, während für $\mathrm{Tl}$ die Inaktivierungsquerschnitte deutlich unter den geometrischen Querschnitten liegen. Dies bedeutet, daß beim $\Phi$ X-174 nahezu jeder Protonendurchgang inaktivierend wirkt ${ }^{7}$, während beim T1-Phagen in der Hälfte aller Fälle 15

21 A. M. Rauth and F. Hutchinson, in: Biological Effects of Ionizing Radiation at the Molecular Level, p. 25, IAEA, Wien 1962.
Primärionisationen ohne schädliche Wirkung bleiben. Übertragen wir unsere bei dem Vergleich der Gammamessungen gefundene Zahl für die Wahrscheinlichkeit einer Reparatur bei $\mathrm{Tl}$ auf die vorliegenden Experimente mit Protonen. Unter den sicher sehr vereinfachenden Annahmen, daß 1. jede Primärionisation einen „Schaden“ erzeugt, daß 2. die Reparaturvorgänge unabhängig voneinander vor sich gehen und daß 3. die Wahrscheinlichkeit der Reparatur eines Schadens 95\% beträgt, so werden 15 Schäden mit einer Wahrscheinlichkeit von $(95 \%)^{15}$ $\cong 46 \%$ repariert. Der mit Protonen gemessene Inaktivierungsquerschnitt sollte folglich etwa $54 \%$ des geometrischen Querschnitts erreichen.

Wir danken Fräulein E. PIetsch, Herrn Dr. Jung und Herrn Priv.Doz. Dr. Hoтz für zahlreiche nützliche Ratschläge und Unterstützung bei der mikrobiologischen Technik, sowie Herrn Kindermann für die Durchführung der Bestrahlungen.

\title{
Analyse der cyclischen Carbonsäure-Dimerisation in Benzol
}

\author{
Kurt Palm \\ Physikalisch-chemisches Laboratorium an der Medizinischen Klinik \\ der Medizinischen Akademie Erfurt \\ (Z. Naturforschg. 22 b, 57-65 [1967]; eingegangen am 29. Juli 1966)
}

\begin{abstract}
Die Substituenten-Abhängigkeit des Dimerisations-Gleichgewichtes von Benzoesäuren und von aliphatischen Carbonsäuren in Benzol wird mittels thermodynamischer Daten $\left(\sigma_{\operatorname{dim}}, \Delta U_{\operatorname{dim}}, \Delta S_{\operatorname{dim}}\right)$ charakterisiert. Sich ergebende Zusammenhänge mit der Protonendissoziation $\left(\sigma_{\text {diss }}\right)$ der Säuren in Wasser und mit ihrer Ionenpaarbildung $\left(\sigma_{\mathrm{ass}}\right)$ mit Diphenylguanidin in Benzol sowie mit den in Tetrachlorkohlenstoff gemessenen infraroten Frequenzlagen $\left(v_{\mathrm{OH}} \mathrm{mon}, v_{\mathrm{CO}} \mathrm{mon}\right.$, dim $)$ weisen darauf hin, daß primär die substitutionsvariable Protonendonator-Fähigkeit der Hydroxylgruppe mit ihrer Solvatations-Wechselwirkung und sekundär der substitutionsveränderte Carbonylakzeptor den Grad der Dimerisation in Benzol bestimmen.
\end{abstract}

Biochemische und molekularbiologische Vorgänge sowie die Molekülstruktur der Nucleinsäuren und Proteine enthalten Wasserstoffbrückenbindungen als strukturbildendes Element. Fortschritte auf diesen Gebieten sind daher mit der Aufklärung von Feinheiten der Wasserstoffbrückenbindung verbunden ${ }^{1}$. Hierzu ist die quantitative Analyse ihrer Struktur und Bindungsenergie an einfachen Systemen mittels experimenteller Methoden unerläßlich. Deshalb wurde die intermolekulare Wechselwirkung an Carbonsäuren, bei denen die Zusammenlagerung zweier

\footnotetext{
1 M. Eigen, Naturwissenschaften 50, 426 [1963].
}

Säuremoleküle über zwei Wasserstoffbrücken zum dimeren Ring gegenüber höherzähligen Assoziaten und gegenüber anderen über Wasserstoffbrücken assoziierenden Stoffen wie Wasser, Alkohole, Phenole stabilisiert ist ${ }^{2 a}$, in einem nichtwäßrigen Lösungsmittel studiert. In dieser Arbeit werden aus thermodynamischen und aus IR-spektroskopischen Messungen gewonnene Ergebnisse zusammenfassend dargestellt und auf der Grundlage der ProtonendonatorProtonenakzeptor-Wechselwirkung gedeutet.

2 H. A. StaAb, Einführung in die theoretische organische Chemie, Verlag Chemie GmbH., Weinheim 1963, a) S. 678, b) S. 602 . 
1. Thermodynamische Parameter

\section{a) Carbonsäure-Dimerisation in Benzol}

Durch Anwendung von Methoden der Kryoskopie, der Ebullioskopie, der isothermen Destillation, der IR-Spektroskopie, der Dielektrik und der Verteilung ergeben sich nach $2(\mathrm{RCOOH}) \rightleftarrows(\mathrm{RCOOH})_{2}$ und $K_{\text {dim }}=\left[(\mathrm{RCOOH})_{2}\right] /[\mathrm{RCOOH}]^{2}$ Dimerisationskonstanten und aus ihrer Temperaturabhängigkeit Dimerisations-Energiewerte. Wegen

$$
K_{\text {dim }}=\exp (-\Delta F / R T) \text { und } \Delta F=\Delta U-T \Delta S
$$

ist auch die freie Energie und die Entropie der Komplexbildung in Lösung berechenbar ${ }^{3,4}$.

Für einige Carbonsäuren in benzolischer Lösung stehen nunmehr zahlreiche Werte zur Verfügung. Diese auf molale oder molare Einheiten bezogenen Daten (Konzentrationsbereich $0,002-0,2 \mathrm{Mol} / l$ ) wurden auf Molenbrüche umgerechnet und $K_{\text {dim }}$ bzw. $\Delta U$ durch Auftragung $\left(\log K_{\text {dim }}\right.$ über $1 / \mathrm{T}$ ) aller Werte neu ermittelt (Tab. 1). Am zuverlässigsten dürften die Daten der ausführlich untersuchten Benzoesäuren (Tab. 1, Nr. 1-9) sein, für die mittlere Fehler von $\log K_{\text {dim }} \pm 2 \%, \Delta U \pm 4 \%, \Delta S \pm 7 \%$ angegeben werden. Weniger genau sind die Werte der aliphatischen Säuren (Nr. 15-22). Die vorhandenen Daten der niederen Fettsäuren (Nr. 10 bis 14) sind nur bedingt verwertbar, da die Hydratation bei Verteilungsmessungen ${ }^{5}$ (vgl. Abb. 3) sowie Anteile höherzähliger Assoziate in die Werte eingehen.

Die Energiewerte sprechen für die cyclische Dimerisation als Hauptassoziationsproze $\beta$, da für die H-Bindung vom $\mathrm{C}=\mathrm{O} \ldots \mathrm{OH}$ Typ im unpolaren Lösungsmittel Werte zwischen -4 bis $-6 \mathrm{kcal} / \mathrm{Mol}$ gelten ${ }^{23}$. Die kleinen $\Delta(F, U, S)$-Beträge bei o-Methoxybenzoesäure sind durch intramolekulare Wasserstoffbrückenbildung bedingt ${ }^{11,35}$. Bezüglich des Substituenteneinflusses zeigt Abb. 1 , daß bei den Benzoesäuren die freie Energie wesentlich vom Substitutionsort $\left|\Delta F_{\mathrm{Bz}, \mathrm{p}}\right|>\left|\Delta F_{\mathrm{m}}\right|>\left|\Delta F_{\mathrm{o}}\right|$ bestimmt wird. Die Substitution beeinflußt Energie und Entro-

3 F. J. C. Rossotri u. H. Rossotri, The Determination of Stability Constants, Academic Press, New York 1961.

${ }^{4}$ H. L. SCHLÄFER, Komplexbildung in Lösung, Springer-Verlag, Berlin 1961.

5 S. D. Christian, H. E. Afrsprung u. S. A. Taylor, J. physic. Chem. 67, 187 [1963].

23 G. C. Pimentel u. A. L. McClellan, The Hydrogen Bond, Reinhold Publ. Corp., New York 1960.

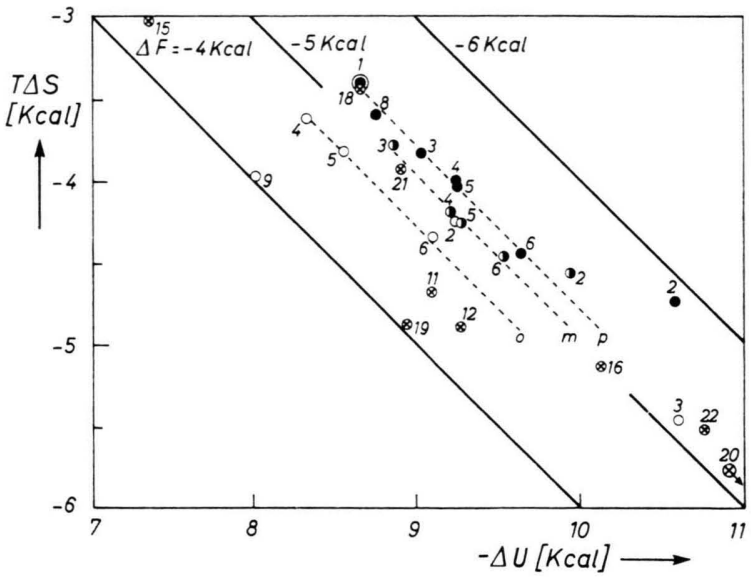

Abb. 1. Entropieänderung $T \Delta S$ in Abhängigkeit von der Dimerisationsenergie $\Delta U$ bei Carbonsäuren in Benzol; Parameter freie Energie $\Delta F$, Bezugstemperatur $25^{\circ} \mathrm{C}$. $\odot$ Benzoesäure, $\bigcirc$ ortho-, meta-, - para-substituierte Derivate, $\oplus$ aliphatische Säuren.

pie, woraus eine funktionelle Abhängigkeit - größere Energieabgabe geht größerer Entropieabnahme bei der Komplexbildung parallel - resultiert, in die sich auch die aliphatischen Säuren einordnen. Zeigen dabei die Daten etwa für $o$-Fluorbenzoesäure bei annähernd gleichem $\Delta F$-Wert eine stärkere Bindung und eine geordnetere Konfiguration des Komplexes an, so behindert die Unsicherheit der Solvatations. anteile $(\Delta U, \Delta S)_{\exp }=(\Delta U, \Delta S)+\left[2(\Delta U, \Delta S)_{\operatorname{mon}}\right.$ - $\left.(\Delta U, \Delta S)_{\text {dim }}\right]_{\text {solv }}$ die quantitative Deutung. Am stärksten wirkt die Solvatation der monomeren Säuremoleküle auf die experimentellen Werte, so daß beispielsweise $\Delta U_{\exp }$ in Lösung weniger negativ ausfällt als in der Gasphase. An Benzoesäure * wurden für $\Delta U_{\text {exp }}$ im Dampfzustand - 13,5 $\mathrm{kcal} / \mathrm{Mol}{ }^{12}$, in Tetrachlorkohlenstoff $-11,3 \mathrm{kcal} /$ $\mathrm{Mol}^{12}$, in Chloroform - 9,4 kcal/Mol ${ }^{24}$ und in Benzol $-8,66 \mathrm{kcal} / \mathrm{Mol}$ bestimmt, wovon in Benzol ca. $+6,0 \mathrm{kcal} / 2 \mathrm{Mol}$ Monomere bzw. ca. - 1,2 kcal/Mol Dimere für die Solvation der Carboxygruppen aufgebracht werden ${ }^{25}$. Meist fehlen jedoch Bezug. werte vom Gaszustand, weshalb die Charakterisierung der Substituenten-Abhängigkeit der Dimerisa-

* G. Allen, J. G. Watkinson u. K. H. WebB, Spectrochim. Acta [London] 22, 807 [1966], weisen an IR-spektroskopisch ermittelten thermodynamischen Werten für die Dimerisation der Benzoesäure in Cyclohexan, Tetrachlorkohlenstoff, Benzol und der Gasphase die gleiche Lösungsmittelabhängigkeit nach.

24 W. MaIER, J. Chim. physique 61, 239 [1964].

25 M. Davies u. J. J. Jones, Trans. Faraday Soc. 50, 1042 [1954]. 


\begin{tabular}{|c|c|c|c|c|c|c|c|c|c|c|}
\hline & & $\sigma_{\text {diss }}$ & Lit. & $\sigma_{\text {ass }^{35}}{ }^{35}$ & $\log K_{\mathrm{dim}}$ & $\sigma_{\mathrm{dim}}$ & $\begin{array}{l}-\Delta F \\
{[\mathrm{kcal}]}\end{array}$ & $\begin{array}{l}-\Delta U \\
{[\mathrm{kcal}]}\end{array}$ & $\frac{-\Delta S}{\left[\frac{\text { cal }}{\text { grd }}\right]}$ & Lit. \\
\hline \multirow{4}{*}{$\begin{array}{l}1 \\
2\end{array}$} & Benzoesäure & 0 & $6,26-30$ & 0 & 3,845 & 0 & 5,25 & 8,66 & 11,5 & $6-13,16,17$ \\
\hline & $o$-Methyl- & 0,26 & 6,30 & $-0,32$ & 3,648 & $-0,197$ & 4,98 & 9,24 & 14,6 & $9,15,16$ \\
\hline & $m$-Methyl- & $-0,07$ & $6,26,30$ & $-0,13$ & 3,919 & 0,074 & 5,35 & 9,93 & 15,4 & $9,15,16$ \\
\hline & $p$-Methyl- & $-0,16$ & $6,26,30$ & $-0,23$ & 4,267 & 0,422 & 5,82 & 10,57 & 15,9 & 6,9 \\
\hline \multirow[t]{3}{*}{3} & $o$-Fluor- & 0,93 & 27 & 0,51 & 3,736 & $-0,109$ & 5,10 & 10,59 & 18,4 & $9-11,14$ \\
\hline & $m$-Flour- & 0,33 & 27 & 0,68 & 3,708 & $-0,137$ & 5,06 & 8,85 & 12,7 & 11,14 \\
\hline & $p$-Fluor- & 0,06 & 27 & 0,35 & 3,806 & $-0,039$ & 5,19 & 9,03 & 12,9 & 11,14 \\
\hline \multirow[t]{3}{*}{4} & $o$-Chlor- & 1,21 & 28,30 & 0,82 & 3,443 & $-0,402$ & 4,69 & 8,32 & 12,2 & $6,9-12,17$ \\
\hline & $m$-Chlor- & 0,37 & 28,30 & 0,80 & 3,674 & $-0,171$ & 5,01 & 9,21 & 14,1 & 11 \\
\hline & $p$-Chlor- & 0,23 & $27,28,30$ & 0,56 & 3,833 & $-0,012$ & 5,23 & 9,24 & 13,5 & 11 \\
\hline \multirow[t]{3}{*}{5} & $o$-Brom- & 1,36 & $27,28,30$ & 0,91 & 3,459 & $-0,386$ & 4,72 & 8,55 & 12,5 & $7,9-11$ \\
\hline & $m$-Brom- & 0,39 & 28,30 & 0,80 & 3,661 & $-0,184$ & 4,99 & 9,26 & 14,3 & 11 \\
\hline & $p$-Brom- & 0,23 & 26,30 & 0,60 & 3,806 & $-0,039$ & 5,19 & 9,22 & 13,5 & 11 \\
\hline \multirow[t]{3}{*}{6} & o-Jod- & 1,31 & 27,30 & 0,96 & 3,473 & $-0,372$ & 4,74 & 9,10 & 14,6 & $9-11$ \\
\hline & m-Jod- & 0,35 & 6,26 & 0,79 & 3,711 & $-0,134$ & 5,06 & 9,53 & 15,0 & 6,11 \\
\hline & $p$-Jod- & 0,22 & 31 & 0,59 & 3,799 & $-0,046$ & 5,18 & 9,63 & 14,9 & 11 \\
\hline \multirow[t]{3}{*}{7} & $0-\mathrm{Oxy}-$ & 1,16 & 28,30 & 2,19 & $3,702 *$ & $-0,047 *$ & & & & 12 \\
\hline & $m-\mathrm{Oxy}-$ & 0,10 & $27,28,30$ & 0,09 & & & & & & \\
\hline & $p-\mathrm{Oxy}-$ & $-0,36$ & $27,28,30$ & $-0,32$ & & & & & & \\
\hline \multirow[t]{3}{*}{8} & $o$-Methoxy- & 0,11 & $\begin{array}{l}27,30 \\
27\end{array}$ & $\begin{array}{r}-1,56 \\
012\end{array}$ & 1,623 & $-2,222$ & 2,21 & 2,06 & 0,0 & 9,11 \\
\hline & $m$-Methoxy- & 0,11 & $\begin{array}{l}27 \\
27\end{array}$ & $\begin{array}{r}0,12 \\
-0,34\end{array}$ & & & & & & \\
\hline & $p$-Methoxy- & $-0,27$ & 27 & $-0,34$ & 3,790 & $-0,055$ & 5,17 & 8,75 & 12,0 & 6,9 \\
\hline \multirow[t]{3}{*}{9} & $o$-Nitro- & 1,77 & 28,30 & 2,18 & 2,954 & $-0,891$ & 4,03 & 8,01 & 13,4 & 9,11 \\
\hline & $m$-Nitro- & 0,71 & $26,27,28$ & 1,56 & & & & & & \\
\hline & $p$-Nitro- & 0,78 & $26,27,28$ & 1,54 & & & & & & \\
\hline 10 & Ameisensäure & 0,45 & $6,32,33$ & & $3,149 *$ & $-0,599 *$ & & & & 13 \\
\hline 11 & Essigsäure & $-0,55$ & 29,33 & & 3,230 & $-0,615$ & 4,41 & 9,10 & 15,7 & $13,18-20$ \\
\hline 12 & Propionsäure & $-0,67$ & 33 & & 3,204 & $-0,641$ & 4,37 & 9,28 & 16,5 & $13,19,21$ \\
\hline 13 & $\operatorname{trans}$-Croton- & $-0,24$ & 6,27 & & $3,887 *$ & $0,138 *$ & & & & 15 \\
\hline 14 & n-Buttersäure & $-0,62$ & 33 & & $3,678 *$ & $-0,071 *$ & & & & 13 \\
\hline 15 & Chloressig- & 1,38 & 6,27 & & 3,114 & $-0,731$ & 4,25 & 7,26 & 10,1 & $13,19,20$ \\
\hline 16 & Phenylessig- & $-0,11$ & 27,29 & & 3,644 & $-0,201$ & 4,97 & 10,12 & 17,3 & $9,15,17,20,22$ \\
\hline 17 & Trimethylessig- & $-0,84$ & 6,27 & & 3,935 & 0,090 & 5,37 & & & 9,13 \\
\hline 18 & Cyclohexancarbon- & $-0,67$ & 27 & & 3,813 & $-0,032$ & 5,20 & 8,65 & 11,6 & 12,22 \\
\hline 19 & Phenylpropiol- & 1,97 & 27 & & 2,971 & $-0,874$ & 4,05 & 8,94 & 16,4 & $6,7,9,20$ \\
\hline 20 & trans-Zimtsäure & $-0,22$ & 27 & & 3,732 & $-0,113$ & 5,10 & 12,10 & 23,5 & $7,9,15,20$ \\
\hline 21 & Hydrozimtsäure & $-0,48$ & 27,29 & & 3,634 & $-0,211$ & 4,96 & 8,90 & 13,2 & $7,9,15,20$ \\
\hline 22 & Stearinsäure & $-0,76$ & 27 & & 3,806 & $-0,039$ & 5,20 & 10,75 & 18,6 & $9,15,17,20$ \\
\hline
\end{tabular}

Tab. 1. Thermodynamische Parameter der Carbonsäure-Dimerisation in Benzol in Relation zur Säuredissoziation in Wasser und zur Ionenpaarbildung mit Diphenylguanidin in Benzol (Bezugstemperatur $25{ }^{\circ} \mathrm{C} \mathrm{bzw} .{ }^{*} 30^{\circ} \mathrm{C}$ ).

6 G. Allen u. E. F. Caldin, Trans. Faraday Soc. 49, 895 [1953]; Chem. Reviews 7, 255 [1953].

7 B. Barton u. C. Kraus, J. Amer. chem. Soc. 73, 4561 [1951].

8 C. R. Bury u. H. O. Jenkins, J. chem. Soc. [London] 1934, 688.

9 H. Dunken u. G. Rudakoff, Z. Chem. 1, 26, 54 [1961].

10 H. Dunken u. K. Palm, Z. Chem. 1, 53 [1961].

11 H. Dunken u. G. Jäger, Z. Chem. 3, 432 [1963].

12 H. Dunken u. Mitarb., unveröffentlichte Meßwerte [1959 bis 1963].

13 H. А. Poнl, M. E. Новвs u. P. M. Gross, J. chem. Physics 9, 408 [1941].

14 A. A. Maryott, M. E. Hobbs u. P. M. Gross, J. chem. Physics 2, 415 [1941].

15 A. A. Maryotr, M. E. Hobrs u. P. M. Gross, J. Amer. chem. Soc. 71, 1671 [1949].

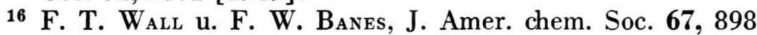
[1945].

17 K. L. Wolf u. G. Metzger, Liebigs Ann. Chem. 563, 157 [1948].
18 M. Davies u. D. M. L. Griffiths, Z. physik. Chem. N. F. 2, 353 [1954].

19 Landolt-Börnstein, Eigenschaften der Materie Bd. II/2, 893 [1960].

20 K. Palm, unveröffentlichte Meßwerte.

21 E. A. Moelwyn-Hughes, D. Patnauk, M. Davies u. P. 0. Jones, J. chem. Soc. [London] 1951, 1249.

22 J. Pirotte, Mèmoire de licence, Univ. Brüssel 1947/48.

26 G. Briegleb u. A. Bieber, Z. Elektrochem., Ber. Bunsenges. physik. Chem. 55, 250 [1951].

27 J. F. J. Dippy, Chem. Reviews 25, 151, 206 [1939].

28 J. F. J. Dippy u. E. A. Hughes, Tetrahedron [London] 19, 1527 [1963].

29 E. J. King u. J. E. Prue, J. chem. Soc. [London] 1961, 275.

30 M. L. Josien, M. D. Peltier u. A. Pichevin, C. R. hebd. Séances Acad. Sci. 250, 1643 [1960].

31 J. M. Vandenbelt, C. Henrich u. S. G. van der Berg, Analytic. Chem. 25, 726 [1954].

32 R. P. Bell u. W. B. Miller, Trans. Faraday Soc. 59, 1147 [1963].

33 Landolt-Börnstein, Eigenschaften der Materie Bd. II/7 a, 847 [1960]. 
tion derzeit auf die qualitative Interpretation der in Lösung ermittelten Werte bei Berücksichtigung von induktiven-, mesomeren-, sterischen- und Solvatationseffekten beschränkt bleibt. Auf dieser Grundlage lassen sich Deutungen für jede Säure finden ${ }^{11,35}$. Dementsprechend ist ein Vergleich der Dimerisation in Benzol mit der Säuredissoziation in Wasser bzw. mit der Ionenassoziation in Benzol sinnvoll.

\section{b) Säuredissoziation in Wasser}

Trotz Unzulänglichkeiten ${ }^{2 b, 26}$ stellen die Dissoziationskonstanten der Säuren $K_{\text {diss }}(\mathrm{Mol} / l)=$ $\left[\mathrm{X}^{\ominus}\right] \cdot\left[\mathrm{H}_{3} \mathrm{O}^{\oplus}\right] /[\mathrm{XH}] \cdot\left[\mathrm{H}_{2} \mathrm{O}\right]$ ein bequemes Mittel zur Kennzeichnung der Strukturabhängigkeit der Protonendonator-Fähigkeit der monomeren Säuremoleküle dar. In Tab. 1 wurden neuere Werte und durch Extrapolation auf extreme Verdünnung gewonnene Konstanten bevorzugt aufgenommen. Dimerisationsanteile in $K_{\text {diss }}$ sind deshalb vernachlässigbar ${ }^{34}$.

\section{c) Ionenpaarbildung mit 1.3-Diphenylguanidin in Benzol}

Für die Assoziation substituierter Benzoesäuren mit der Vergleichsbase 1.3-Diphenylguanidin in Benzol bei $25{ }^{\circ} \mathrm{C}$ ermittelten Davis, Hetzer ${ }^{35}$ Gleich-

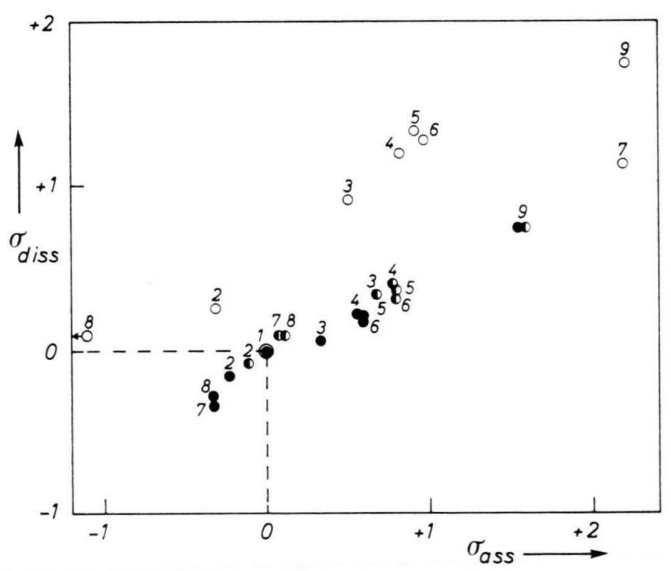

Abb. 2. Parallelität zwischen Protonendissoziation in Wasser $\left(\sigma_{\text {diss }}\right)$ und Assoziation mit 1.3-Diphenylguanidin in Benzol $\left(\sigma_{\text {ass }}\right)$ bei substituierten Benzoesäuren ${ }^{35}$.

34 A. Katchalsky, H. Eisenberg u. S. Lifson, J. Amer. chem. Soc. 73, 5889 [1951].

35 M. M. Davis u. H. B. Hetzer, J. Res. nat. Bur. Standards, A 60, 569 [1958].

36 M. M. Davis u. H. B. Hetzer, J. Amer. chem. Soc. 76, 4247 [1954].

* Die log $K$-Werte einschließlich der Konstanten der aliphatischen Säuren sind auf den jeweiligen Benzoesäurestandard bezogen : gewichtskonstanten $K_{\text {ass }}$. Der Assoziationsvorgang wird entsprechend $\mathrm{Y}+\mathrm{HX} \rightleftarrows \mathrm{YH}^{\oplus} \ldots \mathrm{X}^{\ominus}, \mathrm{K}_{\mathrm{ass}}(l / \mathrm{Mol})$ $=\left[\mathrm{YH}^{\oplus} \ldots \mathrm{X}^{\ominus}\right] /[\mathrm{Y}] \cdot[\mathrm{HX}]$ aufgefaßt als partieller Protonenübergang von der Säure $\mathrm{X}-\mathrm{H}$ zur Base $\mathrm{Y}$ unter Bildung Wasserstoffbrücken-gebundener Ionenpaare ${ }^{36}$. Zwischen $K_{\text {ass }}$ und den ionischen Dissoziationskonstanten der Säuren in Wasser wurde enge Parallelität gefunden, wobei die lineare Abhängigkeit bei $o$-Substitution gegenüber der $m$-bzw. $p$-Substitution differiert (Abb. 2).

\section{d) Der Zusammenhang zwischen $\sigma_{\text {dim }}, \sigma_{\text {diss }}, \sigma_{\text {ass }}$ *}

Die $\left(\sigma_{\text {diss }}, \sigma_{\text {dim }}\right)$-Auftragung (Abb. 3 ) macht den mehrfach erwähnten Zusammenhang ${ }^{6,35,15}$ der freien Energie von Protonendissoziation und Doppelmolekülbildung deutlich: erleichterter Protonen-

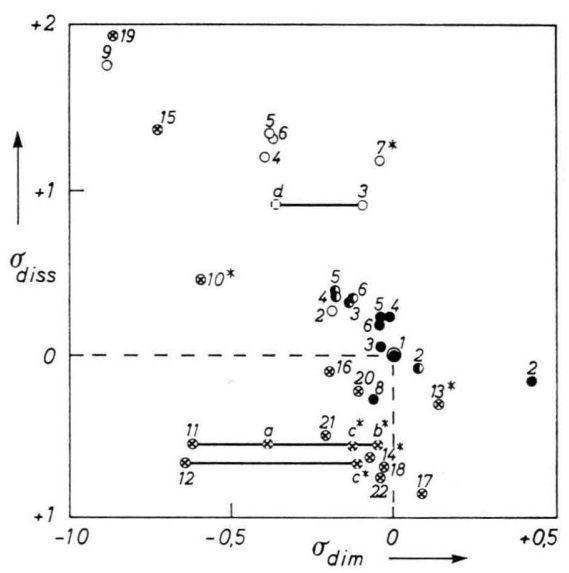

Abb. 3. Zusammenhang zwischen Protonendissoziation in Wasser $\left(\sigma_{\text {diss }}\right)$ und Dimerisation in Benzol $\left(\sigma_{\text {dim }}\right)$ bei Carbonsäuren. Bezugstemperatur $25^{\circ} \mathrm{C}$ bzw. ${ }^{*} 30^{\circ} \mathrm{C}$. Vergleichsweise wurden a) für Essigsäure die aus Verteilungsmessungen unter Berücksichtigung der Monomerhydratation ${ }^{5}$, b) IR-spektroskopisch ermittelte ${ }^{50}$, c) für Essigsäure und Propionsäure aus dielektrischen Messungen gewonnene Dimerisationskonstanten ${ }^{13}$ und d) der aus l. c. ${ }^{10}$ stammende Wert für $o$-Fluorbenzoesäure eingetragen.

dissoziation in wäßriger Lösung steht abnehmende Dimerisation in Benzol gegenüber. Auch die gesättigten Säuren zeigen abgegrenzt von den Benzoesäuren dieses Verhalten. Andererseits folgt aus der $\left(\sigma_{\text {diss }}, \sigma_{\text {ass }}\right)$-Auftragung (Abb.2), daß erleichterter

$$
\begin{array}{cl}
\sigma_{\operatorname{dim}} \equiv \log \left(K_{\mathrm{S}} / K_{\mathrm{Bz}}\right) \operatorname{dim} & \begin{array}{l}
\text { Dimerisationskonstante der sub- } \\
\text { stituierten Säure/Benzoesäure in } \\
\text { Benzol bei } 25^{\circ} \mathrm{C} .
\end{array} \\
\sigma_{\mathrm{ass}} \equiv \log \left(K_{\mathrm{S}} / K_{\mathrm{Bz}}\right) \text { ass } & \begin{array}{l}
\text { Assoziationskonstante der sub- } \\
\text { stituierten Säure/Benzoesäure in }
\end{array} \\
& \begin{array}{l}
\text { Benzol bei } 25^{\circ} \mathrm{C} . \\
\text { Dissoziationskonstante der sub- } \\
\text { stituierten Säure/Benzoesäure in }
\end{array} \\
\sigma_{\text {diss }} \equiv \log \left(K_{\mathrm{S}} / K_{\mathrm{Bz}}\right) \text { diss } &
\end{array}
$$
Benzol bei $25^{\circ} \mathrm{C}$. 
Protonendissoziation in wäßriger Lösung eine leichtere Bildung Wasserstoffbrücken-gebundener Ionenpaare bei der Assoziation mit der Vergleichsbase in Benzol parallel geht.

Dieses Verhalten der Säuremoleküle ist in der Kopplung der intra $\rightarrow$ intermolekularen Wechselwirkung begründet. Dem Protonendonator (Säure) bietet sich als dominierender Protonenakzeptor das Lösungsmittel (bei der Dissoziation in Wasser), die Base (bei der Assoziation mit Diphenylguanidin in Benzol) und die Säure selbst (bei der Dimerisation in Benzol) an. In den ersten beiden Fällen bestimmen substitutionsbedingte intramolekulare Ladungsverschiebungen über die veränderte Protonendonator-Fähigkeit, bei der Dimerisation dagegen über die veränderte Protonendonator- und Protonenakzeptor-Fähigkeit der Säuren den Grad der intermolekularen Wechselwirkung. Dabei steht dem durch Substituenten-Variation beeinflußbaren Donator in den ersten beiden Fällen ein Protonenakzeptor von konstanter, abgestufter Stärke (Wasser, Base und auch Benzol), bei der Dimerisation jedoch auch ein mit der Substitution sich ändernder Protonenakzeptor gegenüber. Trotzdem nun Substitution die Carbonyl- und die Hydroxylgruppe im monomeren Molekül hinsichtlich ihrer intermolekularen Wechselwirkungsfähigkeit entgegengesetzt beeinflußt, indem etwa - I, - M-Substitution Positivierung der $\mathrm{Hy}$ droxylgruppe und Elektronendichte-Abnahme am Carbonylakzeptor bewirkt, resultiert für beide Pole die gleiche Dimerisations-Tendenz in Benzol, da sowohl die verringerte Protonenakzeptor-Fähigkeit der Carbonylgruppe als auch die infolge Positivierung der Hydroxylgruppe verstärkte Solvatation zu abnehmender Dimerisation führen. Dieser Umstand läßt sich an infraroten Absorptionen der Säuren präzisieren.

\section{Die infraroten $v_{\mathrm{OH}}-$ und $v_{\mathrm{OO}}$ - Fre- quenzlagen der Säuren}

An den Säuren wurde die Variabilität der $v_{\mathrm{CO}^{-}}$, $v_{\mathrm{OH}}-$ Frequenz gegenüber Substitution und die der $v_{00}$-Frequenz gegenüber Dimerisation untersucht. $\mathrm{Da}$ die Frequenzen in komplexer Weise vom Lösungsmittel und vom gelösten Stoff abhängen (Carbonsäuren ${ }^{37-39}$ ), wurden vergleichbare Bedingun-

37 A. Allerhand u. P. R. Schleyer, J. Amer. chem. Soc. 85, 371, 1715 [1963].

38 L. C. Bellamy u. R. J. PAce, Spectrochim. Acta 19, 1831 [1963]. gen gewählt: Tetrachlorkohlenstoff (unpolar, kein Protonenakzeptor ${ }^{37}$ ) und teilweise Benzol (unpolar, schwacher Protonenakzeptor ${ }^{37}$ ) als Lösungsmittel, Konzentrationen um $10^{-3} \mathrm{Mol} / l$, Temperatur $30 \pm$ $5{ }^{\circ} \mathrm{C}$. Die Daten gibt Tab. 2 wieder. Bei der Auswertung wurden die aus verschiedenen Arbeiten sowie von eigenen Messungen stammenden Werte auf die jeweilige Benzoesäure-Frequenz normiert.

\section{a) Frequenzen der monomeren Moleküle; Substitu- tions- und Solvatations-Einfluß}

In Tetrachlorkohlenstoff gelöste Benzoesäuren zeigen bei Substitution reziproke Abhängigkeit zwischen $v_{\mathrm{OH}}$ und $v_{\mathrm{CO}}$-Frequenzlage. Einer veränderten $v_{\mathrm{C} O}$-Frequenz entspricht eine gleichgroße, entgegengerichtete $v_{\mathrm{OH}}$-Frequenzverschiebung $\Delta v_{\mathrm{CO}} / \Delta v_{\mathrm{OH}}=$ - 1. Für gesättigte Säuren gilt $\left|\Delta v_{\infty}\right|>\left|\Delta v_{0 \text { Н }}\right|$ (Abb. 4). Die Symmetrie wird zwar maßgeblich von

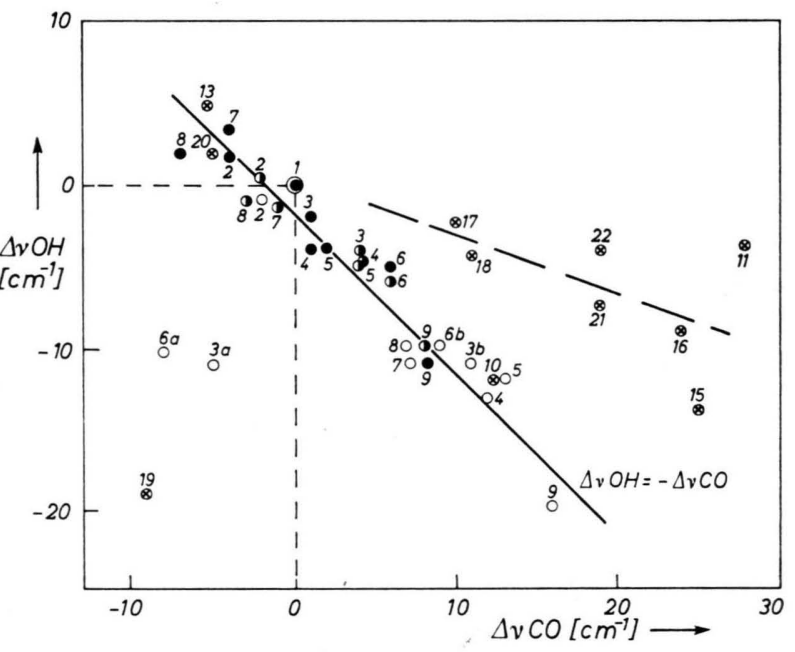

Abb. 4. Die $v_{\mathrm{CO}}$ - und $v_{\mathrm{OH}}$-Frequenzlage monomerer Carbonsäuremoleküle in Tetrachlorkohlenstoff-Lösung (Normierung auf Benzoesäure entsprechend Tab. 2); $a, b$-Konformationsisomere ${ }^{40}$.

Lösungsmitteleigenschaften bestimmt, doch ist diese intramolekulare Kopplung bemerkenswert, da die $v_{\mathrm{CO}}$-Schwingung im Gegensatz zur $v_{\mathrm{OH}}$-Schwingung ziemlich frei von Masse- und von Schwingungskopplungs-Effekten ${ }^{51}$ ist. Mit wenigen Ausnahmen liegen die substitutionsbedingten Frequenzänderungen gegenüber Benzoesäure innerhalb $0-20 \mathrm{~cm}^{-1}$

51 L. C. Bellamy, The IR-Spectra of Complex Molecules, Methuen-Verlag, London 1962, S. 385. 
und nehmen ab mit dem Abstand des Substituenten von der $\mathrm{COOH}$-Gruppe.

Bei den $o$-Halogenbenzoesäuren wurden an der $v_{\mathrm{CO}}$-Bande Komplexisomere unterschieden. Aus dem<smiles>[X]C1CCCCC1C(O)O</smiles>

ca. $1738 \mathrm{~cm}^{-1}$

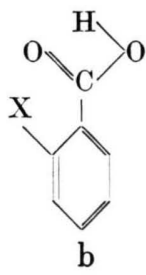

ca. $1755 \mathrm{~cm}^{-1}$
Gang des Intensitätsverhältnisse der beiden Banden von $a / b=0,70$ bei $o$-Fluorbenzoesäure nach $b / a$ $=3,3$ bei $o$-Jodbenzoesäure schloß man, daß die Größe der Halogenatome entscheidend für die Gleichgewichtseinstellung ist, demzufolge das Fluoratom für ein Übergewicht der $a$-Form, das Jodatom für ein Überwiegen der $b$-Form sorgt ${ }^{40}$. Von diesem Effekt abgesehen und unter Berücksichtigung, $\mathrm{da} ß$ vor allem bei konjugierten Systemen die Verknüpfung zwischen Frequenzlage und Molekülstruktur kompliziert und noch unaufgeklärt ist ${ }^{38}$, können näherungsweise die Frequenzverschiebungen als Ausdruck der substitutionsveränderten Elektronenverteilung im monomeren Molekül aufgefaßt werden, die zur Polaritätsbeeinflussung der Carbonyl- und Hydroxylgruppe führt. Wegen dem $\left(v_{\mathrm{CO}}, v_{\mathrm{OH}}\right)$-Zusammenhang (Abb.4) ist dabei der in induktive und mesomere Anteile zerlegbare und durch $\mathrm{Ham}$. met $\mathrm{t}$ - bzw. Taft- Werte charakterisierbare Substituenteneinfluß, schematisch

$$
\underset{(\leftarrow)}{\stackrel{\ominus}{\longrightarrow}} \overbrace{\mathrm{O}-\mathrm{H}_{(-)}^{+} \Delta v_{\mathrm{OH}}}^{\mathrm{O}}
$$

Negativitätszunahme

an beiden Frequenzen ablesbar.

Damit liefern die in Tetrachlorkohlenstoff gemessenen Frequenzen Informationen über die substitutionsveränderte intermolekulare Wechselwirkungsfähigkeit der Säuren. So besteht lineare Abhängigkeit zwischen der $v_{\mathrm{C} 0^{-}}$bzw. $v_{0 \mathrm{H}}$-Frequenzlage der monomeren Moleküle in Tetrachlorkohlenstoff und ihrer Protonenabspaltung in Wasser ${ }^{43,44,30}$ (vgl. Abb. 5) oder mit der Ionenassoziation in Benzol ${ }^{35}$, wobei Säuren mit Mesomerie- bzw. Konjugation zwischen R und $\mathrm{COOH}$ (Benzoesäuren, Croton-,

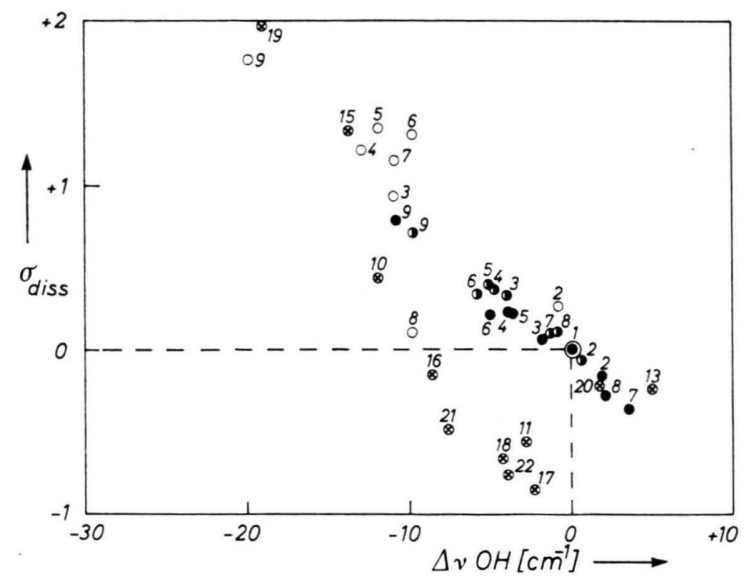

Abb. 5. Zusammenhang zwischen Protonendissoziation in Wasser $\left(\sigma_{\text {diss }}\right)$ und $\nu_{\mathrm{OH}}$ mon-Frequenz in Tetrachlorkohlenstoff bei Carbonsäuren.

Zimt-, Phenylpropiolsäure) gegenüber den gesättigten Säuren differieren. Zum anderen prägt sich auch eine überraschend gute Parallelität zwischen der in Tetrachlorkohlenstoff gefundenen $\nu_{\mathrm{OH}}$-Frequenz der monomeren Moleküle und ihrer Dimerisation in Benzol aus, wodurch der Substituenteneinfluß auf die Dimerisation bereits an der $v_{0 \mathrm{H}}$-Frequenz des monomeren Moleküls voraussagbar wird (Abb.6).

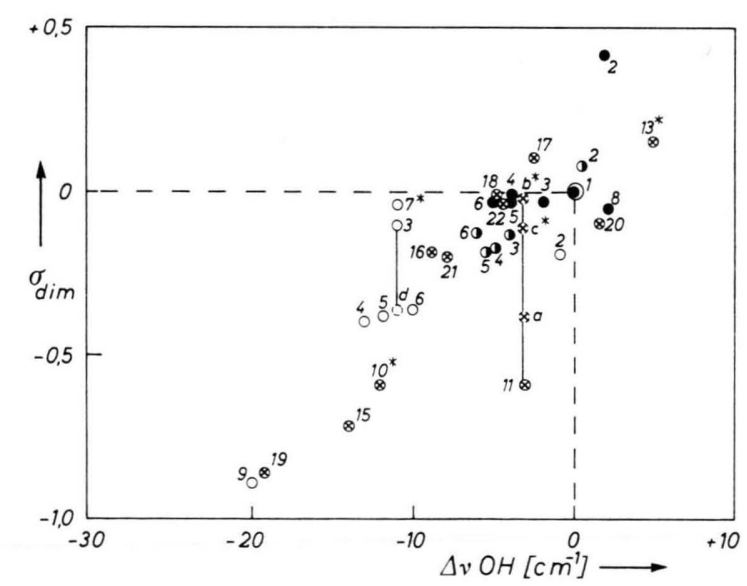

Abb. 6. Zusammenhang zwischen Dimerisation in Benzol $\left(\sigma_{\mathrm{dim}}\right)$ und $v_{\mathrm{OH}}$ mon-Frequenz in Tetrachlorkohlenstoff bei Carbonsäuren.

Als wesentliches Ergebnis erhält man die $\sigma_{\text {diss }}, \sigma_{\mathrm{ass}}$; $\left.\sigma_{\text {dim }^{-}}\right)$Abhängigkeit bestätigt, d. h. daß beispielsweise substitutionsbedingte Positivierung der $\mathrm{Hy}$ droxylgruppe ( $v_{\mathrm{OH}}$-Frequenzabnahme) zur Aciditätserhöhung in Wasser aber zur Dimerisationsabnahme in Benzol führt, wobei zu untersuchen bleibt, 


\begin{tabular}{|c|c|c|c|c|c|c|c|c|c|c|c|c|c|}
\hline & \multicolumn{4}{|c|}{ in Tetrachlorkohlenstoff } & \multicolumn{3}{|c|}{ in Benzol } & \multicolumn{3}{|c|}{ Substitutionseinfluß } & \multirow{2}{*}{\multicolumn{2}{|c|}{$\begin{array}{c}\text { Dimerisations- } \\
\text { einfluß } \\
v_{\text {comon }}-v_{\text {codim }}\end{array}$}} \\
\hline & & Lit. & $\nu_{\mathrm{OH} m o n}$ & $\nu_{\text {Comon }}$ & $v_{\text {codim }}$ & Lit. & $\nu_{\text {Comon }}$ & $v_{\text {Codim }}$ & $\Delta v_{\mathrm{OH} m o n}^{\mathrm{T}}$ & $\Delta v_{\text {COmon }}^{\mathrm{T}}$ & $\Delta \nu_{\text {COmon }}^{\mathrm{B}}$ & & \\
\hline \multirow{6}{*}{1} & Benzoesäure & 30 & ${ }_{37}^{3500+}$ & $\underset{42}{1700+}$ & $1700+$ & 39 & $\underset{45}{1700+}$ & $\underset{-2}{1700+}$ & & & & Tetra & $\begin{array}{c}\text { Benzol } \\
47\end{array}$ \\
\hline & & 40 & 40 & 44 & -3 & 42 & $(25)$ & $(-10)$ & & & & 47 & (35) \\
\hline & & $\begin{array}{l}41 \\
42\end{array}$ & 21 & 41 & -2 & 20 & & & & & & 45 & 42 \\
\hline & & $\begin{array}{l}42 \\
43,44,20\end{array}$ & $\begin{array}{l}45 \\
36\end{array}$ & $\begin{array}{l}37 \\
43\end{array}$ & $\begin{array}{l}-8 \\
-4\end{array}$ & & & & & & & & \\
\hline & & $\begin{array}{l}45 \\
30\end{array}$ & 27 & 40 & & & & & & & & & \\
\hline & 0 -Methyl- & 40 & $\begin{array}{l}37 \\
38\end{array}$ & $\begin{array}{l}40 \\
42\end{array}$ & -4 & 50 & 33 & -11 & $\begin{array}{r}0 \\
-\quad 2\end{array}$ & $\begin{array}{l}-2 \\
-2\end{array}$ & $-9^{c}$ & 46 & 44 \\
\hline \multirow[t]{4}{*}{2} & $m$-Methyl- & 30 & 38 & 40 & $\mathbf{t}$ & 12 & 44 & -3 & $\overline{1}$ & $-\overline{2}$ & $2^{\mathrm{c}}$ & 70 & 47 \\
\hline & $p$-Methyl- & $\begin{array}{l}40 \\
30\end{array}$ & $\begin{array}{l}40 \\
40\end{array}$ & $\begin{array}{l}42 \\
38\end{array}$ & -2 & & & & $\begin{array}{l}0 \\
3\end{array}$ & $\begin{array}{l}-2 \\
-4\end{array}$ & & 44 & \\
\hline & & 40 & 41 & 40 & -3 & & & & 1 & -4 & & 43 & \\
\hline & 0 -Fluor- & $\begin{array}{l}43 \\
40\end{array}$ & Q & 39 & -7 & & & & & -4 & & 46 & \\
\hline \multirow[t]{3}{*}{3} & $\begin{array}{l}o \text {-Fluor- } \\
m \text {-Fluor- }\end{array}$ & 40 & $\begin{array}{l}29 \\
36\end{array}$ & $\begin{array}{c}55,39 \\
48\end{array}$ & $\begin{array}{l}7 \\
3\end{array}$ & $\begin{array}{l}39 \\
12\end{array}$ & $\begin{array}{l}43 \\
42\end{array}$ & $-\frac{2}{2}$ & -11 & 11 & -2 & 48 & 45 \\
\hline & $p$-Fluor- & 40 & 38 & 45 & -1 & 12 & $\begin{array}{l}42 \\
38\end{array}$ & -2 & $\begin{array}{l}-4 \\
-2\end{array}$ & $\begin{array}{l}4 \\
1\end{array}$ & $-2^{\mathrm{d}}$ & $\begin{array}{l}45 \\
44\end{array}$ & $\begin{array}{l}40 \\
40\end{array}$ \\
\hline & $o$-Chlor- & 30 & 27 & 55 & & 39 & 51 & 8 & -10 & 13 & 6 & & 43 \\
\hline & $m$-Chlor- & $\begin{array}{l}40 \\
30\end{array}$ & 27 & 56,38 & 6 & 42 & 17 & -10 & -13 & 12 & $(-8)$ & 50 & (27) \\
\hline 4 & $m$-Chior- & 40 & $\begin{array}{l}32 \\
35\end{array}$ & $\begin{array}{l}40 \\
48\end{array}$ & 3 & 12 & 44 & 2 & $\begin{array}{l}-5 \\
-5\end{array}$ & $\begin{array}{l}3 \\
4\end{array}$ & $4^{\mathrm{d}}$ & 45 & 42 \\
\hline & $p$-Chlor- & 30 & 37 & 45 & & & & & 0 & 3 & & 30 & \\
\hline & & $\begin{array}{l}40 \\
43,44\end{array}$ & $\begin{array}{l}36 \\
35\end{array}$ & $\begin{array}{l}45 \\
46\end{array}$ & $\begin{array}{l}-2 \\
-1\end{array}$ & & & & $\begin{array}{l}-4 \\
-1\end{array}$ & $\begin{array}{l}1 \\
3\end{array}$ & & 47 & \\
\hline & $o$-Brom- & 30 & 25 & 55 & -1 & 39 & 49 & 9 & -12 & 13 & 4 & & 40 \\
\hline \multirow{4}{*}{5} & $m$-Brom- & $\begin{array}{l}40 \\
30\end{array}$ & 28 & $\begin{array}{c}57,38 \\
46\end{array}$ & 11 & 12 & 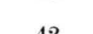 & 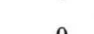 & -12 & 13 & OP & 44 & \\
\hline & $m$-втоm- & 40 & 35 & $\begin{array}{l}40 \\
48\end{array}$ & 2 & 12 & 42 & 0 & -5 & $\begin{array}{l}4 \\
4\end{array}$ & $2^{a}$ & 46 & \\
\hline & $p$-Brom- & $\begin{array}{l}30 \\
40\end{array}$ & $\begin{array}{l}34 \\
36\end{array}$ & 45 & -2 & & & & $\begin{array}{l}-3 \\
-3\end{array}$ & 3 & & 48 & \\
\hline & o-Jod- & 30 & 00 & 52 & $-2+$ & 39 & 50 & 8 & $-\mathbf{t}$ & 10 & 5 & 70 & 42 \\
\hline 6 & m-Jod- & $\begin{array}{l}40 \\
20\end{array}$ & $\begin{array}{l}30 \\
30\end{array}$ & $\begin{array}{c}53,36 \\
49\end{array}$ & $\begin{array}{l}8 \\
2\end{array}$ & 12 & 42 & 0 & $\begin{array}{r}-10 \\
6\end{array}$ & $\begin{array}{l}9 \\
6\end{array}$ & $2^{\mathrm{d}}$ & $\begin{array}{l}45 \\
47\end{array}$ & 42 \\
\hline & $m-50 x^{*}$ & & & & & 20 & 47 & 3 & & & 7 & 76 & $\begin{array}{l}42 \\
44\end{array}$ \\
\hline & $p$-Jod- & $\begin{array}{l}20 \\
30\end{array}$ & $\begin{array}{l}31 \\
25\end{array}$ & 49 & 2 & 20 & 42 & 1 & -5 & 6 & 2 & 47 & 41 \\
\hline & $o-0 x y-$ & 40 & $\begin{array}{c}25 \\
30 \\
\end{array}$ & $\begin{array}{l}-4 \\
-2\end{array}$ & -37 & & & & $\begin{array}{l}-12 \\
-10\end{array}$ & $\begin{array}{l}-46 \\
-46\end{array}$ & & & \\
\hline \multirow[t]{5}{*}{7} & $m-0 x y-$ & $\begin{array}{l}42 \\
30\end{array}$ & $\begin{array}{l}(85) \\
35\end{array}$ & $\begin{array}{l}30 \\
43\end{array}$ & & & & & $\begin{array}{r}(48) \\
-2\end{array}$ & $\begin{array}{l}-7 \\
-1\end{array}$ & & & \\
\hline & $m-0 x y-$ & 44 & 35 & & & & & & $-\overline{1}$ & & & & \\
\hline & $p$-Oxy- & $\begin{array}{l}30 \\
43,44\end{array}$ & $\begin{array}{l}40 \\
40\end{array}$ & $\begin{array}{c}(56) \\
39\end{array}$ & $-3^{20}$ & 20 & 35 & -1 & $\begin{array}{l}3 \\
4\end{array}$ & $\begin{array}{c}(14) \\
-4\end{array}$ & -5 & & 36 \\
\hline & $o$-Methoxy- & & 26 & 50 & & & & & -11 & 8 & & 42 & \\
\hline & & 40 & 30 & 51 & 2 & & & & -10 & 7 & & 49 & \\
\hline 8 & $m$-Methoxy- & 40 & 39 & 41 & -2 & & & & -1 & -3 & & 43 & \\
\hline & $p$-Methoxy- & $\begin{array}{l}40 \\
43,44\end{array}$ & $\begin{array}{l}42 \\
41\end{array}$ & $\begin{array}{l}37 \\
37\end{array}$ & $\begin{array}{l}-9 \\
-9\end{array}$ & & & & $\begin{array}{l}2 \\
5\end{array}$ & $\begin{array}{l}-7 \\
-6\end{array}$ & & $\begin{array}{l}46 \\
46\end{array}$ & \\
\hline & $o$-Nitro- & & 23 & 59 & 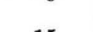 & 12 & 56 & 14 & -14 & 17 & $16^{\mathrm{d}}$ & & 42 \\
\hline & & & 20 & 60 & 15 & & & & -20 & 16 & & 45 & \\
\hline 9 . & $m$-Nitro- & $\begin{array}{l}40 \\
43,44\end{array}$ & $\begin{array}{l}30 \\
28\end{array}$ & $\begin{array}{l}52 \\
50\end{array}$ & $\begin{array}{l}5 \\
9\end{array}$ & & & & $\begin{array}{l}-10 \\
-8\end{array}$ & 8 & & 47 & \\
\hline & $p$-Nitro- & 40 & 29 & 52 & 7 & & & & -11 & 8 & & $\begin{array}{l}41 \\
45\end{array}$ & \\
\hline 10 & Ameisensäure & $\begin{array}{l}44 \\
46\end{array}$ & 27 & 54 & 25 & & & & -9 & 11 & & 99 & \\
\hline 10 & Amersensaure & & 9 & $\begin{array}{l}54 \\
56\end{array}$ & 24 & & & & -12 & 15 & & 39 & \\
\hline 11 & Essigsäure & $\begin{array}{l}12,46 \\
41\end{array}$ & 27 & $\begin{array}{l}71 \\
68\end{array}$ & 14 & 50 & 70 & 27 & & 28 & $28^{c}$ & 57 & 43 \\
\hline & & 47 & 48 & & & & & & $(12)^{\mathrm{a}}$ & 28 & & 50 & \\
\hline & & 48 & 33 & 70 & 16 & & & & -3 & $27^{\mathrm{b}}$ & & 54 & \\
\hline 12 & Propionsäure & $\begin{array}{l}45 \\
20\end{array}$ & & $\begin{array}{l}68 \\
62\end{array}$ & 18 & 20 & 62 & 18 & & $\begin{array}{l}28 \\
19\end{array}$ & 22 & 44 & 44 \\
\hline 13 & trans-Croton- & 20,44 & 41 & 38 & & & & & & & & & \\
\hline $\begin{array}{l}14 \\
15\end{array}$ & $\begin{array}{l}\text { n-Buttersäure } \\
\text { Monochlor- }\end{array}$ & $\begin{array}{l}20 \\
12\end{array}$ & & $\begin{array}{c}61 \\
68,95\end{array}$ & $\begin{array}{l}16 \\
38\end{array}$ & 20 & 59 & 14 & & $\begin{array}{c}18 \\
25,52\end{array}$ & 19 & $30, \frac{45}{57}$ & 45 \\
\hline & essigsä & $\begin{array}{l}46 \\
47\end{array}$ & 39 & 91 & $\begin{array}{l}37 \\
36 \\
\end{array}$ & & & & $(3)^{2}$ & 48 & & 54 & \\
\hline & & 44 & 22 & & $35^{48}$ & & & & -14 & & & & \\
\hline 16 & Phenylessigsäure & $\begin{array}{l}20 \\
44\end{array}$ & 27 & 67 & $\begin{array}{l}15 \\
12^{48}\end{array}$ & $\begin{array}{l}12 \\
20\end{array}$ & $\begin{array}{l}\mathbf{5 5}, \mathbf{7 7} \\
\mathbf{5 2}, 70\end{array}$ & $\begin{array}{l}18 \\
15\end{array}$ & $\begin{array}{l}-9 \\
-9\end{array}$ & 24 & $13,35^{\mathrm{c}}$ & 52 & 37,59 \\
\hline & & 45 & & 60 & & & & & & 20 & & & \\
\hline 17 & Trimethylessigsäure & $\begin{array}{l}20 \\
44\end{array}$ & 34 & 53 & 5 & 20 & 52 & 5 & $\begin{array}{ll}-2 \\
2\end{array}$ & 10 & 12 & 48 & 47 \\
\hline 18 & Cycloh & 20 & $\begin{array}{l}33 \\
32\end{array}$ & 54 & 11 & 20 & 54 & 11 & $\begin{array}{l}-3 \\
-4\end{array}$ & 11 & 14 & $\begin{array}{l}49 \\
43\end{array}$ & 43 \\
\hline 19 & $\begin{array}{l}\text { carbonsäure } \\
\text { Phenylpropiolsäure }\end{array}$ & $\begin{array}{l}44 \\
20\end{array}$ & $\begin{array}{l}31 \\
17\end{array}$ & 34 & -9 & 20 & 30 & -10 & $\begin{array}{r}-5 \\
-19\end{array}$ & -9 & -10 & 43 & 40 \\
\hline 10 & Pnenyipropioisaure & 44 & 17 & & -5 & & & -10 & -19 & -5 & -10 & 70 & \\
\hline 20 & trans-Zimtsäure & $\begin{array}{l}20 \\
44\end{array}$ & $\begin{array}{l}38 \\
38\end{array}$ & 38 & -2 & 20 & 31 & -6 & $\begin{array}{l}2 \\
2\end{array}$ & -5 & -9 & 40 & 37 \\
\hline & & 45 & & 28 & & & & & & -12 & & & \\
\hline 21 & Hydrozimtsäure & 20 & 28 & 62 & 15 & 12 & 58 & 13 & -8 & 19 & $16^{\mathrm{c}}$ & 47 & 45 \\
\hline & & $\begin{array}{l}44 \\
45\end{array}$ & 29 & 56 & & & 64 & 17 & -7 & 16 & & & \\
\hline 22 & Stearinsäure & 20 & 32 & 62 & 13 & 20 & 64 & 14 & -4 & 19 & 24 & 49 & 50 \\
\hline
\end{tabular}

Tab. 2. Die $\nu_{\mathrm{OH}}$ und $v_{\mathrm{CO}}$-Frequenzen von Carbonsäuren; Substitutions- und Dimerisations-Einfluß. a) auf Benzoesäure $3536 \mathrm{~cm}^{-1}$, b) $1743 \mathrm{~cm}^{-1}$, c) $1742 \mathrm{~cm}^{-1}$, d) $1740 \mathrm{~cm}^{-1}$ bezogen, () unwahrscheinliche Zuordnung. Den Substituenteneinfluß auf die Frequenzen der monomeren Moleküle gibt $\Delta \nu_{\mathrm{OH}, \mathrm{T}, \mathrm{Co}}=\left(\nu_{\text {Säure }}-v_{\text {Benzoesäure }}\right)$ wieder. 
ob die stärkere Solvatationswechselwirkung der Hydroxylgruppe mit Benzol $\triangle \mathrm{R}-\mathrm{COOH} \ldots \longrightarrow$ oder die verringerte Akzeptorfähigkeit der Carbonylgruppe $\left(v_{\mathrm{CO}}\right.$-Frequenzzunahme $)$ entscheidenden Einfluß auf die Dimerisation ausüben.

Beim Lösungsmittelwechsel Tetrachlorkohlenstoff $\rightarrow$ Benzol tendiert die $v_{\mathrm{C} O}$-Frequenz gesättigter Säuren um ca. $4 \mathrm{~cm}^{-1}$ nach höheren, die ungesättigten Säuren um den gleichen Betrag nach niederen Werten. Gegenüber der konstant bleibenden Frequenz der Benzoesäure zeigen die $o$-Halogenbenzoesäuren Frequenzabnahmen um ca. $6 \mathrm{~cm}^{-1}$, wobei $o$-Fluorbenzoesäure mit $1755,1738 \rightarrow 1743 \mathrm{~cm}^{-1}$ auffällt. $\mathrm{Da}$ in Benzol bei den $o$-Halogenbenzoesäuren keine Aufspaltung der Carbonylbande auftritt ${ }^{12}$ und im kristallinen Zustand die $b$-Form ${ }^{52-54}$ und zudem bei $o$-Fluorbenzoesäure eine nebenvalente Wechselwirkung zwischen dem Fluoratom und dem benachbarten Benzolwasserstoff von ca. $1 \mathrm{kcal} / \mathrm{Mol}$ nachgewiesen wurde ${ }^{54}$, dominiert offenbar - übereinstimmend mit den Dipolmomenten der Säuren in Ben-

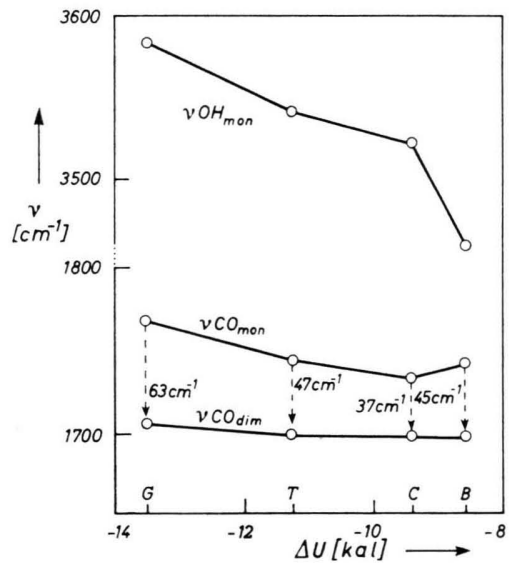

Abb. 7. Solvatations-Beeinflussung der IR-Frequenzen $v_{\mathrm{OH}}$ mon, $v_{\mathrm{CO} \text { mon }}, v_{\mathrm{CO}}$ dim und der Dimerisationsenergie $\Delta U_{\text {exp }}$ von Benzoesäure in Tetrachlorkohlenstoff ${ }^{12}$, in Chloroform ${ }^{24}$ und in Benzol gegenüber der Gasphase ${ }^{12}$.

${ }^{39} \mathrm{~K}$. PALM, Optik und Spektroskopie aller Wellenlängen, Akademie-Verlag, Berlin 1962, S. 512.

40 C. J. W. Brooks, G. Eglington u. J. F. Morgan, J. chem. Soc. [London] 1961, 106, 661 .

41 S. Bratoz, D. Hadzi u. N. Sheppard, Spectrochim. Acta [London] 8, 249 [1956].

42 P. E. Murray u. S. Sundaram, Canad. J. Chem. 39, 1625 [1961].

43 S. C. Flett, Trans. Faraday Soc. 44, 767 [1948].

44 J. D. S. Goulden, Spectrochim. Acta [London] 6, 129 [1954].

45 A. J. Collings u. K. L. Morgan, J. chem. Soc. [London] 1963, 3437.

46 J. Bellanto u. I. R. Barcelo, Spectrochim. Acta [London] 16, 1333 [1960]. zol $^{55}$ - die für die Solvatation der Hydroxylgruppe günstige $b$-Form. Die starke Solvationswechselwirkung der Hydroxylgruppe wird durch $\boldsymbol{v}_{\mathrm{OH}}$-Frequenzabnahmen um ca. $80 \mathrm{~cm}^{-139}$ beim Lösungsmittelwechsel bestätigt. Am Beispiel der Benzoesäure kann der Solvatationseinfluß auf die $v_{0 \mathrm{H}}$-Frequenz und auf die in demselben Lösungsmittel bestimmte Dimerisationsenergie direkt demonstriert werden (Abb. 7).

\section{b) Frequenzänderungen durch Dimerisation}

Dimerisation verändert die $v_{\mathrm{CO}}$ - und $v_{\mathrm{OH}}$-Frequenz des monomeren Carbonsäuremoleküls um einen Betrag, der weit über den beobachteten Substitutionsund Lösungsmittel-bedingten Frequenzverschiebungen liegt.. Da die Carbonylfrequenz des Dimeren den Substitutionseinfluß vom monomeren Molekül direkt widerspiegelt, resultiert für alle Benzoesäuren in Tetrachlorkohlenstoff - und offenbar auch in Benzol (vgl. Tab. 2) eine Substitutionsunabhängige konstante Dimerisations-Verschiebung $v_{\mathrm{CO} \text { mon }}-\boldsymbol{v}_{\mathrm{CO} \operatorname{dim}}=(45 \pm 5) \mathrm{cm}^{-1}$. Den gleichen Wert liefern die aliphatischen Säuren, ausgenommen Ameisen- $\left(30 \mathrm{~cm}^{-1}\right)$, Essig- $\left(55 \mathrm{~cm}^{-1}\right)$ und Chloressigsäure $\left(30,55 \mathrm{~cm}^{-1}\right)$, d. h. Säuren, welche stark zur Kettenassoziation neigen. Die DimerisationsVerschiebung zeigt zwar Lösungsmittelabhängigkeit, welche durch die Solvatation der monomeren Moleküle bedingt ist (Abb. 7), jedoch lassen sich keine Aussagen über substitutionsbedingte DimerisationsÄnderungen gewinnen. Offenbar charakterisiert der substitutionsunabhängige $\quad v_{\mathrm{CO}}$-Dimerisationsshift einen konstanten energetischen Beitrag des Akzeptoratoms zur Wasserstoffbrückenbindung. Für die intensive $v_{\mathrm{OH}}$-Absorption dimerisierter Moleküle ein breites Absorptionsband zwischen 3000-2500 $\mathrm{cm}^{-1}$ mit Submaxima - liegen hinsichtlich der Substituenten-Abhängigkeit noch keine Ergebnisse vor.

47 J. T. Harris u. M. E. Нobbs, J. Amer. chem. Soc. 76, 1419 [1954].

48 L. C. Bellamy, R. F. Lake u. R. J. Pace, Spectrochim. Acta [London] 19, 443 [1963].

49 R. H. Gillette, J. Amer. chem. Soc. 58, 1143 [1936].

50 D. P. N. Scatchell u. J. L. Wardell, Trans. Faraday Soc. 61, 1199 [1965].

52 G. Ferguson u. G. A. Sim, Acta crystallogr. [Copenhagen] 14, 1262 [1961].

53 G. Ferguson u. G. A. Sim, Acta crystallogr. [Copenhagen] 15, 346 [1962].

54 J. Krausse u. H. Dunken, Acta crystallogr. [Copenhagen] 20, 67 [1966]. 


\section{Schlußbetrachtung}

Beim zweipoligen intermolekularen Wechselwirkungstyp $\mathrm{R}-\mathrm{COOH}$... A (Wasser, Base) modifiziert Substitution einen Pol - die ProtonendonatorFähigkeit der Hydroxylgruppe - wodurch sich eindeutige Substituenten-Abhängigkeiten ergeben (vgl. Abbn. 2 und 5). Diese prägen sich bei der Dimerisation der Carbonsäuren in Benzol schwächer aus (vgl. Abbn. 3 und 6), da Substitution sowohl im dimeren Molekül die Carbonyl- und die Hydroxylgruppen $\Delta \mathrm{R}-\mathrm{C}\left\langle\begin{array}{l}\mathrm{O} \ldots \mathrm{HO} \\ \mathrm{OH} \ldots \mathrm{O}\end{array}\right\rangle \mathrm{C}-\Delta \mathrm{R}$ als auch beim monomeren Molekül die Hydroxylgruppe und damit die Solvatations-Wechselwirkung $\mathrm{R}-\mathrm{COOH}$... A (Benzol beeinflußt (Abschn. $1 \mathrm{~d})$.

Die Parallelität (Abb.6) zwischen substitutionsbedingter Dimerisationsabnahme (-zunahme) und $\nu_{\mathrm{OH}}$-Frequenzerniedrigung (-erhöhung) läßt keine Entscheidung zu, ob Dimerisationsabnahme von der infolge Positivierung der Hydroxylgruppe $\left(v_{\mathrm{OH}}-\right.$ Frequenzabnahme) stärkeren Solvatation oder von der verringerten Protonenakzeptor-Fähigkeit der Carbonylgruppe $\left(v_{\mathrm{C} O}\right.$-Frequenzzunahme) dominierend bestimmt wird. Jedoch sind der substitutionsunabhängige Dimerisationsshift und die aus dielektrischen Polarisationsmessungen sich ergebende konstante

55 K. PALM, Z. physik. Chem., in Vorbereitung.
Excesspolarisation der dimeren Moleküle ${ }^{55}$ Anzeichen für einen schwachen Substituenteneinfluß auf den dimeren Ring, so da $\beta$ in Verbindung mit der $\left(v_{\mathrm{OH}}, v_{\mathrm{CO}}, \Delta U\right)$-Abhängigkeit (Abb. 7) der Schluß naheliegt, daß bei den untersuchten Carbonsäuren primär die substitutionsvariable ProtonendonatorFähigkeit der OH-Gruppe mit ihrer SolvatationsWechselwirkung und sekundär der substitutionsveränderte Carbonylakzeptor den Grad der Dimerisation in Benzol bestimmen. Dies steht in Einklang mit der Säurefunktion der Moleküle als auch mit der $\mathrm{C}$ a $\mathrm{n} n$ o n schen Untersuchung ${ }^{56}$, daß im Vergleich mit der Amid $\mathrm{C}=0$-Gruppe der Carbonylgruppe der Säuren geringere Polarität, jedoch ihrer $\mathrm{OH}$ Bindung größere Polarität gegenüber der Amid NH-Bindung zukommt. In Konsequenz dieser Deutung ist für den Substituenteneinfluß bei Eliminierung der Solvatation - im Dampfzustand - eine veränderte Substituenten-Abhängigkeit der Carbonsäure-Dimerisation gegenüber der in Benzol gefundenen zu erwarten.

Herrn Prof. Dr. A. Sundermann - Direktor der Medizinischen Klinik Erfurt - sowie Herrn Prof. Dr. H. Dunken und Herrn Dipl.Chem. Dr. G. JäGER vom Institut für Physikalische Chemie der Universität Jena möchte ich für die Förderung dieser Arbeit besonders danken.

56 C. G. Cannon, cit. in: Ergebn. exakt. Naturwiss. 31, 270 [1959]. 